\title{
Structural Changes in Spleen Architecture upon Plasmodium berghei (NK-65) Infection in BALB/c Mice
}

\author{
Vineet kumar ${ }^{1}$ and Upma Bagai ${ }^{1}$ \\ Parasitology Laboratory, Department of Zoology, Panjab University, Chandigarh-160014, India ${ }^{1}$
}

\begin{abstract}
Malaria, caused by an apicomplexan parasite Plasmodium, is a major cause of morbidity and mortality throughout the world. In present study, Plasmodium berghei (NK-65) was found to be lethal to $B A L B / c$ strain of Swiss white mice, when injected parasitized erythrocytes. Malaria infection has been reported to induce acute injuries to vital organs i.e. liver, kidney and spleen of infected host. The spleen is the largest secondary immune organ in the body and is responsible for initiating immune reactions to blood-borne antigens and for filtering the blood of foreign material and damaged red blood cells. All the important functions are carried out by two main compartment of spleen: white and red pulp. Splenomegaly was observed with rise in infection. Haematoxylin/Eosin stained transverse sections of normal mouse spleen showed an intact capsular region with trabecule emanating into splenic parenchyma. Spleen of post-infection mice exhibited disturbed splenic architecture enlarged white pulp area, infected cells, sinusoidal dilation, haemozoin deposition and transient loss of marginal zone due to P.berghei infection.
\end{abstract}

Keywords: Haemozoin, malaria, Plasmodium, spleen, splenomegaly.

\section{Introduction}

Malaria is one of the most common vector-borne diseases of tropical and subtropical regions of the world. Globally, an estimated 3.3 billion people were at risk of malaria in 2011, with populations living in subSaharan Africa having the highest risk of malaria infection. Approximately half of countries with ongoing malaria transmission are on track to meet the World Health Assembly target to achieve a $75 \%$ reduction in malaria case incidence rates by 2015, compared to levels in 2000 [1]. Chemotherapy and vector control programs have been largely ineffective due to the emergence and spread of insecticide resistant mosquito vectors and drug resistant. A completely effective vaccine must contain not one but most of the molecule involved in different stages of parasite invasion of host cells [2]. The development of a safe, effective and affordable malaria vaccine is a critical global public health priority [3].

Malaria infection has been reported to induce acute injuries to vital organs. The most pronounced changes inflicted due to disease involve in blood, spleen, liver and kidney of infected host. Efficient control of pathogens by the immune system is promoted by a highly organized microarchitecture of secondary lymphoid organs. These structures form the basis for trapping, transport, processing, and presentation of antigens (Ags), a prerequisite for initial constraint of pathogens and successful induction of specific immunity. The spleen is a complex, largest secondary lymphoid organ that is perfectly adapted to selectively filtering and destroying senescent red blood cells (RBCs), infectious microorganisms and Plasmodium-parasitized RBCs. Infection by malaria is the most common cause of spleen rupture and splenomegaly, albeit variably, a landmark of malaria infection. Present study has been designed to evaluate the structural and histological changes occurring in spleen of mice due to plasmodial infection.

\section{Materials And Methods}

\subsection{Animal model and ethical clearance:}

Five to six weeks old inbred BALB/c mice of either sex, weighing 15-20 g, were used as experimental animals for present study. All animals were purchased from Institute of Microbial Technology (IMTECH), Chandigarh. Animals were kept in ventilated cages in Central Animal House, Panjab University, Chandigarh. They were fed with standard feed and water ad libitum. The care and handling of animals as well as other procedures were strictly performed following guidelines approved by the Institutional Animal Ethics Committee (45/99/CPCSEA) of the Panjab University, Chandigarh.

\subsection{Parasite and infection:}

Asexual erythrocytic stages of $P$. berghei (NK-65) strain were maintained in BALB/c mice. Infection were initiated by intra-peritoneal (i.p) inoculation of $1 \times 10^{6}$ infected erythrocytes obtained from infected to naïve mice. The course of infection was monitored by Giemsa- stained thin blood films [4]. 


\subsection{Light microscopy:}

The animals were anaesthetized and spleen was excised and processed for light microscopic study. For light microscopy the tissue was fixed in Bouin's fixative for 5-6 hrs and kept in water overnight. The tissues were dehydrated in various grades of alcohol and embedded in paraffin wax with melting point (M.P.) of 58$60^{\circ} \mathrm{C}$. Five microns thick paraffin sections were stained with hematooxyline and eosin stain. The sections were mounted in DPX and observed under light microscope under suitable magnification [5].

\section{Results}

For morphological and histopathological studies, mice of normal, $P$. berghei infected groups were sacrificed after light anesthesia with sodium pentathol. Spleens were extracted out from normal and infected group at low (D3) and high infection (D7). These were washed properly in $0.9 \%$ saline solution and tissues were fixed in Bouin's fixative for overnight.

\subsection{Morphological changes in spleen}

$P$. berghei infected mice were dissected on day 3 and 7 post-inoculation. Spleens were taken out and weighed after washing with saline. The normal mice spleen weight was $(0.09 \pm 0.02 \mathrm{~g})$ and it was dark red, located in the abdomen just below the diaphragm at the posterior end of the pancreas behind stomach. As the parasitaemia increased, the weight of spleen also increased. Splenomegaly was evident due to rise in infection. At low infection $(6.33 \pm 2.9 \%)$, weight of spleen was $0.35 \pm 0.12 \mathrm{~g}$ and slight enlargement of spleen was observed. At high infection $(33.9 \pm 6.1 \%)$, the size of spleen was significantly increased $(0.78 \pm 0.25 \mathrm{~g})$. The color of infected spleen was dark brown due to congestion and deposition of haemozoin pigment.

\subsection{Histopathological changes in spleen}

\subsubsection{Normal spleen}

Light microscopic examination of transverse sections (T.S.) of normal spleen stained with haematoxylin-eosin revealed intact capsule of connective tissue outside the tissue (Fig.3.2.1: A). From inside of capsule, a rich network of trabeculae sub-divided the organ into communicating compartments (Fig. 3.2.1: D). The tissue enclosed within the capsule is reticular connective tissue. The splenic pulp was observed to be divided into two zones i.e. red pulp and white pulp (Fig.3.2.1: A). The red pulp is made up of splenic sinuses/sinusoids and splenic cords (Fig. 3.2.1: D). Splenic cords lie between the sinuses, separating them from one another. The reticular cells are present in the splenic sinuses of red pulp. The blood cells like macrophages are found in sinuses, as are plasma cells. The splenic cords contained lymphocytes and monocytes (T-cells) in high concentration (Fig. 3.2.1: B, C).

White pulp contained lymphocytes, plasma cells, macrophages and reticulum cells. The white pulp is divisible into two components i.e. periarteriolar lymphatic sheath (PALS) and lymphatic follicles. PALS appeared as cylinders which coaxially surround central artery. It consists of meshwork of reticulum cells and extracellular reticulum. Marginal zone lie between the red pulp and white pulp appeared as junctional tissue. It consists of reticular cells, vascular space, lymphocytes, plasma cells, macrophages. The lymphocytes appeared as small nucleated cells while plasma cells and macrophages as large cells. Normal erythrocytes appear in red pulp area (Fig. 3.2.1: B, C, E, F).

\subsubsection{Spleen at low infection (D3)}

Transverse sections of $P$. berghei infected spleen at low infection (1.5-2.0\%) on D3 in (GP-II) stained with Haematoxylin-eosin stain revealed the altered splenic architecture due to indistinct appearance of red and white pulp in low infection. The marginal zone was transiently lost (Fig. 3.2.2: A). White pulp hyperplasia was observed along with reduced PALS area. Significant congestion of both RBCs and parasitized erythrocyte (PE) was also noted in red pulp region (Fig. 3.2.2: B, D). The presence of haemozoin pigment and sinusoidal dilations were commonly observed in infected spleen sections. Deposition of haemozoin pigment was evident in red pulp (Fig. 3.2.2: C). White pulp (WP) subdivided into marginal zone, follicles and periarteriolar sheath (PALS) was observed (Fig. 3.2.2: D). Lymphocytes, macrophages, reticular cells were observed in white pulp (Fig.3.2.2: B, E). Concentric layers of reticular fibers and flattened reticular cells were observed in PALS region. Mild dilation of trabeculae, proliferation of lymphocytes, monocytes and reticulum cells were observed in white pulp region. Parasitized and normal erythrocytes along with mild sinus dilation were observed in red pulp (Fig. 3.2.2: E, F).

\subsubsection{Spleen at high infection (D7)}

Infected red cells were visible in red and white pulp area at high infection (35-40\%) in haematoxylin and eosin stained sections. Complete distortion of marginal zone at high infection was observed (Fig. 3.2.3: A). 
The red pulp was observed to be expanded markedly by venous congestion, haematopoiesis (Fig, 3.2.3: B, C). Reticular connective tissue consists of reticular cells were also observed in red pulp and white pulp. However, reticular cells appeared long tapered rod like cells without branching in red pulp regions but these were branched in white pulp region (Fig. 3.2.3: D). Haemozin pigmentation and number of macrophages were more prominent (Fig.3.2.3: B, C, D, F). Venous sinus dilation and vacuolization of white pulp and red pulp along with parasitized erythrocytes were observed. Thickening of capsule and destruction of trabeculae was observed at high infection (Fig. 3.2.3: D, E, F). Beneath the capsule moderate proliferation of lymphocytes and monocytes was observed.
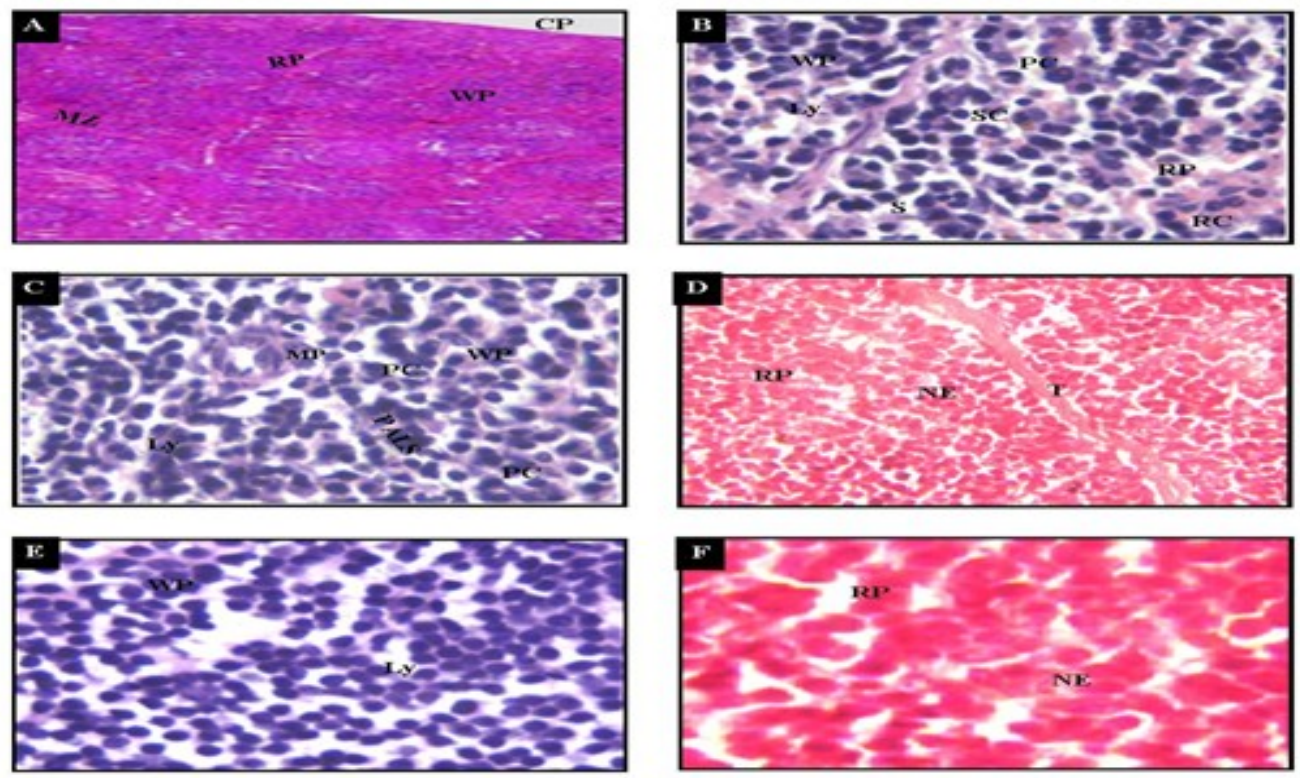

Figure 3.2.1: Transverse sections of normal spleen of BALB/c mouse stained with Haematoxylin/eosin [A-(4X), B, C \& D-(40X) E, F (100X)] CP-Capsule, MZ-Marginal zone, WP-White pulp, RP-Red pulp, NE-Normal erythrocyte, S-Sinusoid, Ly- Lymphocyte, MP-Macrophage, M- Monocyte, RC-Reticular cell, SC-Splenic cord, PCPlasma cell, PALS- Periarteriolar lymphatic sheath.
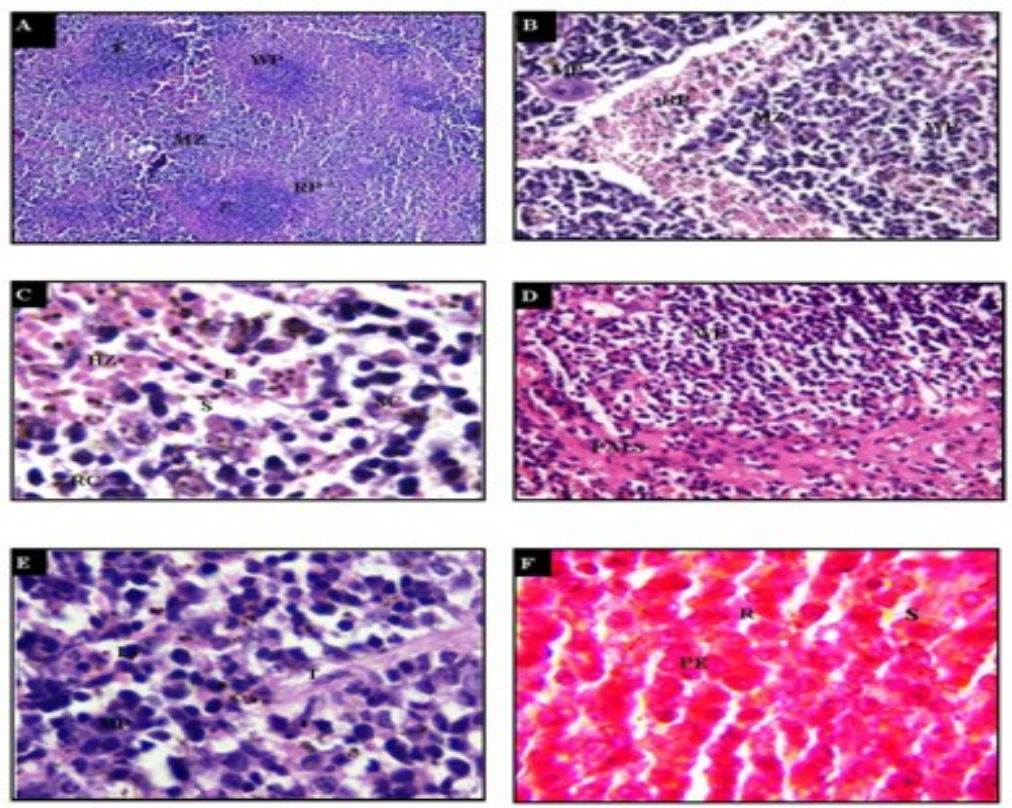

Fig. 3.2.2: Transverse sections of $P$. berghed infected spleen (GP-II) of BALE/c mouse at low infection (1.5-2.0\%), (D3) (stained with Haematoxylin/eosin [A-(20x), B, C \& D-(40X), E,F (100X)] CP-Capsule, T-Trabeculae, MZ-Marginal zone, WP-White pulp, RP-Red pulp, WPWhite pulp, PE-Parasitized erythrocyte, R-Red cell, S-Sinus, Ly-Lymphocyte, PALSPeriarteriolar bymphatic sheath, HZ-Haemozoin pigment, RC-Reticular cell, MPMacrophage, E-Erythrocyte. 

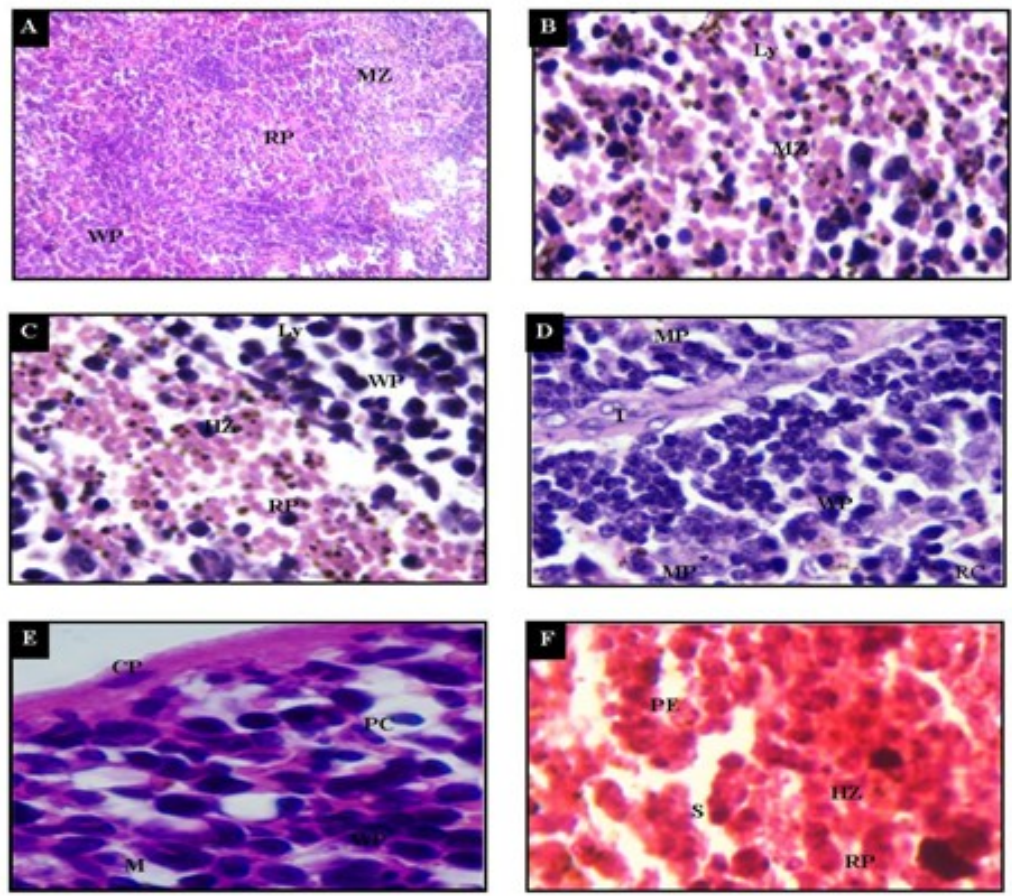

Figure 3.2.3: Transverse sections of $P$. berghei infected spleen (GP-II) of BALB/c mouse at high infection (35-40\%), (D7) stained with Haematoxylin/eosin [A-(10X), B, C-(40X) \& D, E, F-(100X)] CP-Capsule, T-Trabeculae, MZ-Marginal zone, WP-White pulp, RP-Red pulp, MP-White blood cell, R-Red cell, S-Shizont, M-Monocyte, MPMacrophage, HZ-Haemozoin pigment, PE-Parasitized erythrocyte, PC-Plasma cell, Ly-Lymphocyte.

\section{Discussion}

Rodent malaria parasites have been widely used as valuable models for various experimental purposes due their adaptability to the laboratory conditions. Swiss white mice Mus musculus (BALB/c strain) have been confirmed to be susceptible to $P$. berghei infection. Different workers have reported the lethality of $P$. berghei to mice [6,7]. Inoculation of parasitized erythrocytes to naïve mice results in $100 \%$ lethal infection [8]. Size of the inoculum can influence the progress of murine malaria in the host but $P$. berghei infection has been reported to be invariably fatal in mice [9]. P. berghei has a asynchronous life cycle in mice and invades prefentially reticulocytes [10]. Most of the normal mice when injected with $1 \times 10^{6} P$. berghei, died because of high infection by the day 7 or 8 post inoculation except those in which parasite migrated to reticulocytes followed by increase in parasitaemia till death of mice. Janse et al. (1989) have also reported preferential invasion of $P$. berghei merozoites in the reticulocytes in vitro [11]. The Histopathological studies on the spleen were performed in normal, $P$. berghei infected, groups. In normal spleen red pulp and white pulp areas were clearly evident. The splenic capsule was intact, trabeculae conataining artery were distinct. Splenic sinuses/sinusoids and splenic cords were observed including reticular cells, macrophages, T-cells (lymphocytes and monocytes) and plasma cells in normal mice.

In present study, splenomegaly is the most common sign of malaria infection was observed. Size of spleen increases several fold after infection, due to influx of lymphocytes in both human and mouse infection $[12,13]$. Malaria associated splenomegaly has been reported due to increased erythrocytes in mouse spleen $[14,15]$. Loss of marginal zone was observed with increase in parasitemia. Loss of marginal zones and general loss of follicle in spleen has been reported in P. chabaudi infection [16]. Proliferative changes in P. berghei infected spleen were observed due to white pulp hyperplasia which is associated with reduction in the PALS area but an increase in B-cell follicle and germinal centre areas. Similar observation were made by many investigator $[17,18]$. Accumulation of haemozoin pigment has been reported to occur due to protein degradation by parasite [19]. The present finding also indicates accumulation of haemozoin and infiltration of red blood cells in sinusoidal spaces in infected spleen.

Other studies reported that marginal zone macrophages (MZM) and marginal metallophillic macrophages (MMM) are lost from spleen following infection with P. c. chabaudi AS [20]. Reticular cells were observed in normal as well as infected spleen. Studies carried out out by other workers have described the modification of reticular cell networks in the red pulp during murine $P$. yoelli infection to form a barrier that channels blood directly from artery to the vein, thereby preventing access to red pulp macrophages [21]. Parasitized erythrocytes were observed with increase in infection in red pulp at high infection on D7. The spleen 
is a key site for removal of parasitized red blood cells, generation of immunity and production of new red blood cells during malaria. Extrafollicular foci of B-cell plasma cells were observed in PALS following P. c. chabaudi infection [22]. Expansion of white pulp, marginal zone and red pulp were the major changes that occurs in the spleen during murine malaria infection which point towards to the is erythropoiesis and hematopoiesis and similar investigation were reported by other workers [20,23].

\section{CONCLUSION}

Spleen is an important organ involved in generation of immune response against pathogens. The spleen seems to have an important role in both controlling and establishing chronic malaria infection, because of its unique architecture designed to allow coordination of its phagocytic and cellular immune mechanisms. Understanding of these important mechanisms require the exact knowledge about interactions among various cell types occuring in the spleen. Further studies on spleen are required as many mechanisms are still unclear about generation of immunity during malaria infection and upon immunization.

\section{ACKNOWLEDGMENTS}

Mr. Vineet Kumar is thankful to University Grant Commission, New Delhi for providing financial assistance under UGC-Major Research project .

\section{REFERENCES}

[1]. World Health Organization, World Malaria Report, 2013.ISBN 9789241564674

[2]. EP Manuel, and AM. Patarroyo, Emerging rules for subunit based multi antigenic multistage chemically synthesized vaccines, Acc Chem Res, 41, 2008, 377-86.

[3]. EM. Malkin, DJ. Diemert, JH. McArthur, JR. Perreault, AP. Miles, BK. Giersing, GE. Mullen, A. Orcutt, O. Muratova, M. Awkal, H. Zhou, J. Wang, A. Stowers, CA. Long, S. Mahanty, LH. Miller, A. Saul, and AP. Durbin, Phase 1 clinical trial of apical membrane antigen 1: an asexual blood-stage vaccine for Plasmodium falciparum malaria, Infection and Immunology, 73, 2005, 3677-3685.

[4]. U. Bagai, and A. Pawar, A blood stage fraction of Plasmodium berghei induces protective and long lasting immune response in BALB/c mice, Parasitology International, 62, 2013, 329-336.

[5]. A.G.E. Pearse, Carbhohydrates. In. histochemistry: theoretical and applied (revised edition of A.G.E. Pearse). Churchill A and Churchill J (eds), London. 1968, 228-280.

[6]. LH. Miller, and R. Carter, Innate resistance in malaria. Experimental Parasitology, 40, 1976, 132-146.

[7]. U. Bagai, Immunological studies on some antigens from asexual erythrocytic stages of rodent malaria parasite, P. berghei. Ph.D. Thesis, Panjab University, Chandigarh, 1992.

[8]. HC. Pedroni, CC. Bettoni, SM. Spalding, and TD Costa, Plasmodium berghei: Development of an irreversible experimental malaria model in wistar rats, Experimental Parasitology, 113 (3), 2006,193-196.

[9]. A. Martinelli, B. Franke-Fayard, D. Djokovic, M. Dooren, W, J. Ramesar, AP. Waters, M. Falade, O. Kranendonk, M. Cravo, P. Janse, Simple and sensitive antimalarial drug screening in vitro and in vivo using transgenic luciferase expressing Plasmodium berghei parasites, International Journal of Parasitol. 38(14), 1651-1662.

[10]. CJ. Janse, EG, Boorsma, J. Ramesar, MJ. Grobbee, and B. Hons, Host cell specificity and schizogony of $P$. berghei under different in vitro conditions, International Journal of Parasitology, 19, 1989, 509-514.

[11]. MD. Dakhel, Studies on some antigens from asexual erythrocytic stages of Plasmodium berghei. Ph. D. Thesis, Panjab University, Chandigarh, 2001.

[12]. WR. Pitney, The tropical splenomegaly syndrome, Transaction of Royal Socitey of Tropical Medicine and Hygiene, 62(5), 1968, 717-728

[13]. L. Weiss, J. Johnson, and W. Weidanz, Mechanisms of splenic control of murine malaria: tissue culture studies of the erythropoietic interplay of spleen, bone marrow, and blood in lethal (strain 17X) Plasmodium yoelii malaria in BALB/c, American Journal of Tropical Medicine and Hygiene, 41, 1989, 135-143.

[14]. S. Tsubata, K. Ebe, T. Kawamura, Y. Ishimoto, C. Tomiyama-Miyaji, H. Watanabe, H. Sekikawa,Y. Aoyagi, and T. Abo, Protection against malaria by anti-erythropoitic antibody due o suppression of erythropoiesis in the liver and at other sites, Journal of Immunology and Cell Biology, 83, 2005, 638-642.

[15]. D.Nogueira, R. Ron-EI, S. Friedler, M. Schachter, A. Raziel, and R. Cortvrindt, Meitic arrest in vitro by phosphodiesterase 3-inhibitir enhances maturation capacity of human oocytes and allows subsequent embryonic development, Biological Reproduction, 74, 2006, 177-184.

[16]. ET. Cadman, AY. Abdallh, C. Voisine, AM. Sponaas, P. Corran, T. Lamb, D. brown, F. ndungu, and j. Langhorne, Alterations of splenic architecture in malaria are induced independently of Toll-like receptors 2,4 and 9 or MyD88 and may affect antibody affinity, Infection and Immunity, 76((9), 2008, 3924-3931.

[17]. A. Sophiagena, and S. Frankenburg, Lymphocyte and mmacrophages dynamics in spleen of BALB/c mice in course of infection, after rechallenge of cured mice, Experimental Parasitiology, 65, 1988, 50-60.

[18]. AD. Sullivan, I. Ittarat, and SR. meshmick, Patterns of haemozoin accumulation in tissue, Parasitology, 112(3), 1996, 285-294.

[19]. AH. Achtman, M. Khan,IC. MacLennan, and J. Langhorne, Plasmodium chabaudi chabaudi infection in mice induces strong B cell responses and striking but temporary changes in splenic cell distribution, Journal of Immunology, 171, 2003, 317-324.

[20]. H. Helmby, G, Jonsson, M. Troye-Blomberg, Cellular changes and apoptosis in spleen and peripheral blood of mice infected with blood-stage Plasmodium chabaudi chabaudi AS, Infection and Immunity, 2000; 68: 1485-9.

[21]. MM. Stevenson, and G. Kraal, Histological changes in the spleen and liver of C57BL/6 and A/J mice during Plasmodium chabaudi AS infection, Experimental and Molecular Pathology, 1989,51:80-95.

[22]. HJ. Alves, W. Weidanz, and L. Weiss, The spleen in murine Plasmodium chabaudi adami malaria: stromal cells, T lymphocytes, and hematopoiesis, American Journal of Tropical . Medicine and Hygiene ., 55, 1996, 370-378.

[23]. RR. Freeman, and CR. Parish, Spleen cell changes during fatal and self-limiting malarial infections of mice, Immunology., 35, $1978,479-484$. 